Aus der Universitäts-Frauenklinik in Königsberg i. Pr. (Direktor: Prof. Dr. Winter.)

\section{Spontane Uterusruptur im Beginne der Geburt.)}

\section{Von Dr. H. Gans, Assistenzarzt.}

Die spontanen Rupturen des Uterus in der Gravidität sowohl als auch im Beginne der Geburt sind so seltene Ereignisse, die Frage ihrer Entstehung noch immer eine so strittige, daB ein jeder derartige Fall der Mitteilung wert erscheint.

Aus diesem Grunde will ich über folgende, in klinischer wie in pathologischer Hinsicht exakt angestellte Beobachtung ausführlich berichten:

Anamnese: Patientin, eine 33 jährige Arbeiterfrau, ist früher nie ernstlich krank gewesen. Erste Periode mit 14 Jahren, seitdem Menses anfangs regelmäßig alle vier Wochen, vom 16.-22. Lebensjahr alle sechs bis sieben Wochen, nach dem zweiten Partus wieder alle vier Wochen, stets von neun- bis elftägiger Dauer, schwach, mit spickenden Kreuzschmerzen und diffusen Unterleibsschmerzen verbunden; vom dritten Tage ab war Patientin schmerzfrei, an den ersten beiden Tagen bettlägerig. Letzte Menses 12. Mai 1907. Patientin hat vier Partus, einen Abort durchgemacht. Sämtliche Entbindungen spontan, ohne ärztliche Hilfe. Kinder lebend geboren. Nachgeburtsperioden bei der ersten Geburt normal, bei der zweiten bis vierten erschwerte Expression durch die Hebamme. Die Wochenbetten verliefen ohne Besonderheiten; am neunten Tage stand $\mathrm{Pa}$ tientin auf. Im August 1905 Abort von sieben Wochen. Ursache unbekannt. Spontaner Verlauf nach mehrtägiger, mäßiger Blıtung. Wegen danach anhaltender, mäßig starker, konstanter Blutungen suchte Patientin nach etwa sieben Wochen die hiesige gynäkolo. gische Poliklinik auf. Befund: Uterus etwas vergrößert, dextrovertiert, etwas weich, deutliches Hegarsches Zeichen. Temperatur $37,8^{\circ}$. Diagnose: Retentio post abortum. Therapie: Kurettement in Narkose. Vom Tage nach der Ausschabung ab hohes Fieber und viele Schüttelfröste; es bestand Meteorismus und starke Schmerzhaftigkeit des ganzen Abdomens; vom vierten Tage ab häufiges Erbrechen. Sehr reichlicher, übelriechender Fluor. Drei Wochen lang Eisbehandlung, Opiate, später warme Umschläge. Erst nach drei Monaten stand Patientin auf und erholte sich langsam; sie fühlte sich wohl und war völlig beschwerdefrei. Ausfluß bestand nur eine Woche vor der Periode; er war wei $B$, nicht übelriechend, nicht ätzend.

Die Menses traten nach dem Abott alle vier Wochen, nur die ersten drei Male in fünf- bis sechswöchigen Intervallen auf; sie waren von neun- bis elftägiger Dauer, aber etwas stärker als früher. Die dysmenorrhoischen Schmerzen blieben die gleichen. Am 12. Mai 1907 hatte Patientin die letzte Regel. Abgesehen von den üblichen Schwangerschaftsbeschwerden, Uebelkeit, Erbrechen etc., verlief die Gravidität zunächst ungestört. Ende September 1907 begannen Kindsbewegungen. Während dieselben bei früheren Schwangerschaften schmerzlos waren, hatte Patientin bei dieser Gravidität von Anfang der Bewegungen an bei jeder Lokomotion des Kindes sehr starke, spickende Schmerzen in der rechten Unterleibsseite; die Bewegungen selbst waren mäßig stark und traten mehrmals am Tage auf. Bestanden anfangs die Schmerzen nur bei den Kindsbewegungen, so traten sie in gleicher Intensität und von gleichem Charakter bald auch ohne solche auf; zuweilen beim Anftreten, beim Liegen auf der rechten oder linken Seite, oft bei völliger Bettruhe. Sie betrafen stets nur die rechte Unterleibsseite, hatten meist krampfartigen Charakter und hielten jedesmal etwa ein bis zwei Minuten an; sie waren niemals besonders stark. Andere Erscheinungen, Meteorismus, Fiebergefühl, Erbrechen, fehlten; keine Blutungen. Der Leibesumfang entsprach dem bei früheren $\mathrm{Ge}_{-}$ burten.

Am 20. Januar 1908, 11 Uhr a. m. begannen schwache, wehenartige, besonders in der rechten Unterleibsseite lokalisierte Schmerzen. die in Abständen von $1 / 2$ Stunde einander folgten. Gleichzeitig bestand ein starkes Frostgefühl. Die hinzugezogene Hebamme fand die Lage gut, eine Oeffnung kaum vorhanden; sie verließ wegen geringen Fortgangs der Geburt die Kreißende. 8 Uhr p. in. be.

1) Im Auszug vorgetragen im Verein für wissenschaftliche Heilkunde, Königs. berg i. PI, 25. Mai 1908 . gann Patientin zu erbrechen, das Erbrechen wiederholte sich bis heute Mittag (21. Januar) noch 10-12 mal. Seltenes AufstoBen trat auf. $9 \mathrm{Uhr}$ p. m. Die Wehen folgen alle 15 Minuten. Von nun an bestanden aber nicht nur mehr Wehen, sondern zwischen denselben, von den Wehen sicher unterscheidbar, traten starke rechtseitige, krampfartige Unterleibsschmerzen auf. Diese wurden allmählich immer stärker, sodaß Patientin vor Schmerzen zuweilen laut aufschreien mußte. Seit Wehenbeginn lag Patientin zu Bett. $11 \mathrm{Uhr}$ p. m. fand die Hebamme bei ihrer Untersuchung den Muttermund knapp für einen Finger durchgängig. Die Wehen blieben gleich stark, traten in Zwischenräumen von etwa $1 / 4$ Stunde auf, zwischen den fast konstanten rechtseitigen Unterleibsschmerzen erkennbar. Patientin hatte bei den Schmerzen rechts das Gefühl, als ob alles wund sei. 12 Uhr nachts bekam Patientin plötzlich das Gefühl, als ob etwas in ihrem Leib platze. Ohnmacht oder Schwindel trat nicht ein. Von nun an begann der Leib allmählich etwas dicker zu werden. Die Kindsbewegungen hielten, wenn auch schwach, nach dem Platzen noch an; um 1 Uhr a. m. sistierten sie völlig. Bald nach $12 \mathrm{Uhr}$ hatte die Hebamme noch schwache, stark verlangsamte Herztöne gehört, am Morgen nicht mehr; in der Zwischenzeit wurde nicht auskultier亡. Nach dem Platzen wurden die Schmerzen immer größer, zeitweise unerträglich. Die Wehen hielten, wenn auch schwächer und in großen Intervallen, bis heute $11 \mathrm{Uhr}$ a. m. an. Um $5 \mathrm{Uhr}$ a. m. untersuchte die Hebamme ein drittes Mal; sie fand jetzt wieder alles geschlossen. Nunmehr wurde Patientin zur Beschleunigung der Geburt gebadet (10 Minuten lang, ziemlich warm.) Um $8 \mathrm{Uhr}$ a. $\mathrm{m}$. begannen die rechtseitigen Schmerzen etwras nachzulassen. Im Laufe des Vormittags wurde Patientin immer elender, zuweilen bestand leichter Schwindel und Flimmern vor den Augen, zunehmende Blässe. Keine Blutung nach außen. Blasensprung nicht beobachtet. Gegen Morgen stellten sich leichte Dyspnoe und Stiche in der Brust ein, starkes Herzklopfen. Wegen des mangelnden Fortschrittes der Geburt und des schlechten Allgemeinbefindens wurde poliklinische Hilfe erbeten, die um $11 \mathrm{Uhr}$ a. m. eintraf und auf Grund der Diagnose - Uterusruptur - die sofortige Einlieferung in die Klinik anordnete.

Status. Mittelgroße, kräftig gebaute Frau. Haut und sichtbare Schleimhäute blaß. Lungen ohne Besonderheiten. Herz: Grenzen normal; Spitzensto $B$ an normaler Stelle. Lautes, systolisches Geräusch über allen Ostien. Zweite Töne rein, nicht akzen tuiert. Puls schwach fühlbar, 116, regelmäßig. Sensorium frei. Haut fühlt sich kühl an. Abdomen mäßig aufgetrieben, überall empfindlich; in beiden Lendengegenden Dämpfung, Fluktuation.

Rechts von der Mittellinie fühlt man bis zum Rippenbogen hinauf sehr deutlich dicht unter den Bauchdecken liegende Kindsteile. Herztöne nicht zu hören. Links, drei Querfinger unterhalb des Nabels, ein rundlich erscheinender Tumor, der als Uterus an. gesprochen wird.

Vaginaler Befund. Kein Abgang von Blut. Scheide ohne Besonderheiten. Cervix völlig erhalten, gerade für einen Finger durchgängig.

Der durch die Cervix geschobene Finger fühlt links liegend den Uterus entsprechend der von außen gefühlten Resistenz und palpiert in ihm nach rechts hin einen kleinen Kindsteil.

Nach diesem Befund wird die anfangs auf Extrauteringravidität gestellte Diagnose fallen gelassen und an eine in ihrer Aetiologie zunächst unklare Uterusruptur gedacht.

Operation: (Operateur Prof. Winter.) Desinfektion der Bauchdecken. Hautdecken dünn. Nach Durchschneiden der Fascie wird das Peritoneum vorgewölbt, durch welches blauschwarzes Blut durchschimmert. Nach Eröffnung des Peritoneums müssen die stark sich vortreibenden, geblähten Darmschlingen teilweise eventriert werden, wobei sich massenhaft Blutgerinnsel entleeren. Ganz leicht lösbare Verwachsungen des peritonealen Uterusüberzuges mit der Umgebung. Nachdem nunmehr Uebersicht gewonnen ist, wird das rechts unter den Därmen versteckte, frei in der Bauchhöhle liegende Kind nach Durchtrennung der Nabelschnur entfernt und auch die jetzt sichtbar werdende Placenta samt den Eihäuten, die sich obenfalls ganz aus dem Uterus entleert haben, herausgenommen. Aus dem schlaffen Fruchtsack entleert sich dabei reichlich gelbliches, klares Fruchtwasser. Erst jetzt sieht man, daß es sich um eine Tterusruptur handelt. Der gut kontrahierte Uterus wird mit Zangen vorgezogen und weist eine vom Fundus nach rechts hinten ziehende, bis zur Höhe des inneren Muttermundes reichende, breite, komplette Ruptur mit rissigen, morschen Rändern auf. Bei dieser Sachlage wird von einer konservativen Operation vorn vornherein abgesehen und sofort die supravaginale Amputation in Aussicht genommen. Abbinden der Ligamenta rotunda und der Adnexe beiderseits am Uterus. Umschneiden der oberen Blasengrenze und $\mathrm{Zu}$ rückpräparieren der Blase; diese erweist sich als intakt. Abbinden der Arteria uterina auf beiden Seiten. Bei dem Versuch, dieses rechts auszuführen, zeigt sich, daß hier die Arterie in der Rißstelle 
liegt; sie wird zunächst hier nicht aufgefunden. Ohne daß das Peritoneum hinten umschnitten und zurückgeschoben wird, erfolgt die Amputation des Uterus in Höhe des Os internum; dabei trifft der Schnitt die unteren Ausläufer des Risses. Nach Anziehen der rechten Cervixkante mit einer Kugelzange wird eine Umstechung, etwas tiefer als links, unter Mitfassen der Cervixkante um die rechte Uterina gelegt. Der Stumpf ist blutfrei; er wird durch fünf Catgutknopfnähte exakt verschlossen, worauf das Peritoneum in querer Richtung, von einem Adnexstumpf zum anderen hinüber, über dem Cervixstumpf vernäht wird.

Nunmehr werden die Därme möglichst von den Blutgerinnseln, die ihnen anliegen, befreit und in die Bauchhöhle zurückgebracht. Schluß der Bauchdecken in drei Etagen - Peritoneum, Fascie, Haut. - Der Gesamtblutverlust muß, zumal da das bei der Beckenhochlagerung in die Zwerchfellkuppe geflossene Blut nicht zu Gesicht gekommen ist, auf $2-2 \frac{1}{2}$ Liter geschätzt werden.

Zu Beginn der Operation Infusion von $1000 \mathrm{ccm}$ Kochsalzlösung, drei Spritzen Kampfer. Von Beginn der Beckenhochlagerung wird der bis dahin kaum fühlbare Puls besser und bleibt auch während der ganzen Operation leidlich gefüllt, gut fühlbar, 120-140.

Die Rekonvaleszenz verlief völlig reaktionslos. 32 Tage nach der Operation wird Patientin beschwerdefrei, bei durchaus gutem Allgemeinbefinden entlassen.

Anatomischer Befund: (siehe die Abbildung.) Der stark kontrahierte Uterus weist an seiner Vorderfläche und an der Hinter

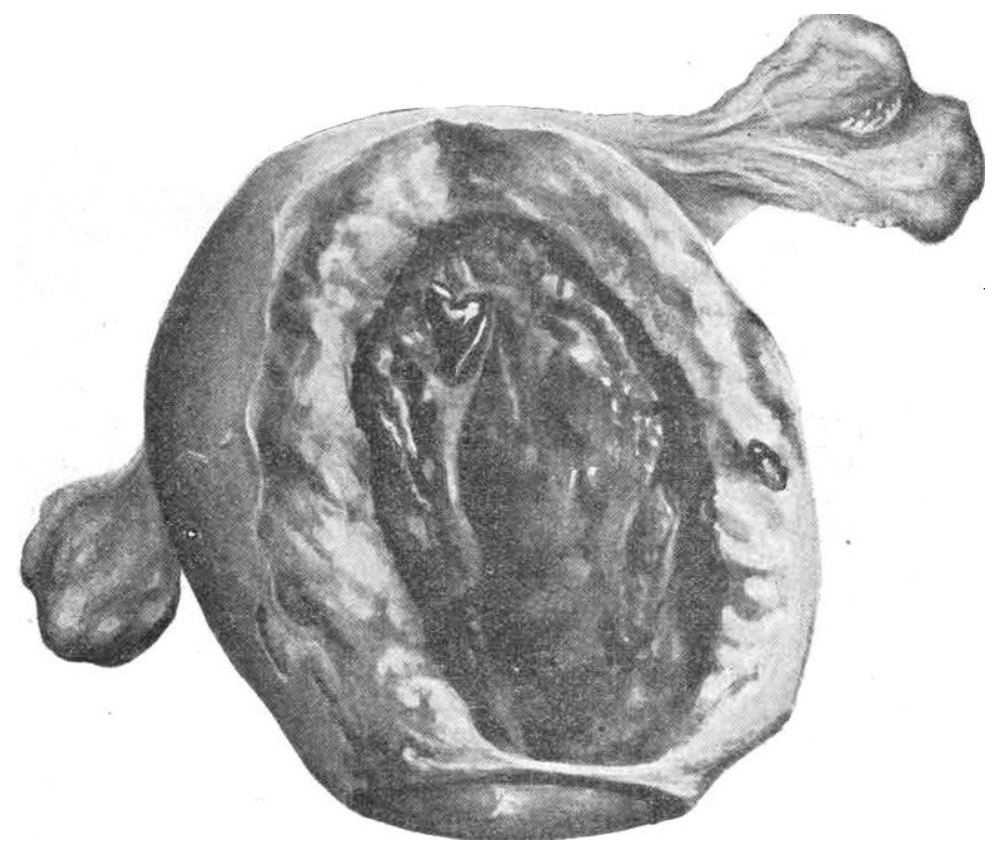

Ansicht von hinten.

Spontane Uterusruptur bei einer 33 făhrigen V.-Gebărenden inı Beginn der Geburt. Kind und Placenta in die Bauchhŏhle ausgetreten.

fläche vereinzelte flächenhafte Adhäsionen auf. Die Adnexe sind mit ihrer Umgebung durch breite Verwachsungen verbunden.

Auf der Hinterfläche des Uterus ist, $2 \mathrm{~cm}$ unterhalb des rechten Tubenansatzes beginnend, ein Riß sichtbar, der sich von dort aus $10,5 \mathrm{~cm}$ weit durch die ganze Hinterwand gerade nach unten verlaufend erstreckt. Die Ränder dieses Risses sind stark nach auBen gekrempelt, sodaß es sich bei dem stark kontrahierten Zustand des Uterus nicht ermöglichen läßt, die beiden Ränder aneinander zu bringen. Die freien Rißränder sind nicht scharf, sondern uneben, etwas zerfetzt. Das Peritoneum ist von den Rändern etwas zurückgeschoben und gleichfalls zerfetzt und zerfranzt. Der Abstand der beiden inneren Ränder ist nahe dem Fundus $4 \mathrm{~cm}$, in der Mitte $5 \mathrm{~cm}$, und an der Amputationsfläche $5,5 \mathrm{~cm}$. Der Abstand der äußeren Ränder beträgt nahe dem Fundus $6 \mathrm{~cm}$, in der Mitte $9 \mathrm{~cm}$, und an der Amputationsstelle $8 \mathrm{~cm}$. Die Breite der Ränder, d. $\mathrm{h}$ die Dicke der an der Rißstelle freiliegenden Muskulatur der hinteren Uteruswand, ist überall gleichmäßig, etwa $2 \frac{1}{2} \mathrm{~cm}$. Weder hier noch sonst am Uterus findet sich eine Verdünnung der Muskulatur. Die freiliegende Muskulatur sieht dunkelrot aus und ist teilweise mit platten, lose aufsitzenden, frischen Blutgerinnseln belegt. Die Uterushöhle reicht, von der Amputationsfläche an gerechnet, $9,5 \mathrm{~cm}$ weit. Sie ist austapeziert mit frischen, geronnenen Blutmassen.

Die Ansatzstelle an der Placenta scheint an der Rißstelle zu liegen, doch läßt sich dies durch die fest aufliegenden Blutcoagula mit Bestimmtheit makroskopisch nicht feststellen. An der Amputationsfläche findet sich ein doppelstricknadeldicker Strang, der beide Ränder miteinander verbindet, ein Beweis, daß die Amputation unterhalb des Risses vorgenommen wurde.

Die linkseitige Tube ist von normaler Dicke, die Fimbrien gut ausgebildet, das abdominale Ende offen, die Tube in ganzer Ausdehnung bequem durchgängig. Das linke Ovarium von normaler Größe weist ebenso wie die linke Tube Adhäsionen auf. Auch die rechte Tube ist für die Sonde passierbar, normal dick. Das walnußgroße rechte Ovarium weist ein Corpus luteum auf. Auch an den rechten Adnexen finden sicb. Adhäsionen.

Der Abstand der beiderseitigen Ligamenta rotunda - das rechte setzt etwas höher an als das linke - beträgt $83 / 4 \mathrm{~cm}$.

Das männliche Kind ist frischtot, ohne Zeichen von Mazeration, $50 \mathrm{~cm}$ lang, $2570 \mathrm{~g}$ schwer. Seine Kopfmaße betragen:

$$
\begin{aligned}
& \text { Diam. fronto-occipitale }: 11 \mathrm{~cm} \\
& \text { mento-occipitale }: 12,5 \\
& \text { biparietale } \\
& \text { bitemporale } \\
& \text { Kopfumfang } \\
& \begin{array}{l}
9,5 \\
: 7,5
\end{array}
\end{aligned}
$$$$
\text { : } 33,5 \text {, }
$$

Die Placenta, $390 \mathrm{~g}$ schwer, ist etwa doppelthandflächengrol3 gleichmäBig entwickelt mit geringer Cotyledonenbildung. Makroskopisch Gewebe ohne Besonderheiten, nur am Rande ein zweimarkstückgroßer Infarct. Die Decidua etwas verdickt. Die Eihäute von normalem Aussehen sind in halber Ausdehnung dicht am Rande abgerissen. Die Nabelschnur setzt zentral an, ist dick und sulzreich, $59 \mathrm{~cm}$ lang.

Mikroskopischer Befund: Mikroskopische Schnitte wurden in großer Anzahl aus verschiedenen Stellen des Uterus hergestellt, sowohl von der Rißstelle als auch von anderen Partien der Uteruswand. Das Material zur mikroskopischen Untersuchung wurde in Zelloidin eingebettet, jedoch wurden auch Gefrierschnitte von nicht eingebettetem Material untersucht. Diese letzteren ergaben nichts anderes als die Zelloidinpräparate. Vor allem konnte an ihnen nachgewiesen werden, daß eine fettige Degeneration der Uterusmuskulatur nicht vorlag. Gefärbt wurden die Schnitte mit Hämatoxylin, Hämatoxylin Eosin, van Gieson und Orcein (Unna-Ta enzersche Vethode). In den Schnitten von der Rißstelle sind Zotten nur in geringer Menge vorhanden. Ein Wuchern derselben in die Uteruswand ist nicht zu bemerken. Die Zottenstruktur ist völlig normal. Das Syncytium ist intensiv gefärbt; das Stroma hat gut färbbare Kerne. Syncytiale Wanderzellen dringen von den Zotten aus in ganz geringer Weite in das Gewebe vor. An der Muskulatur findet sich keine Veränderung. Ueberall dicke Muskelbündel mit gut färbbaren Kernen und regelmäBigen Konturen. Das Bindegewebe auf den nach van Gieson gefärbten Schnitten scharf hervortretend; an keiner Stelle - besonders auch auf keinem Schnitt aus dem Bereich des Risses - vermehrt. In der Muskulatur sieht man an den Schnitten der Rißstelle kleinere und größere Blutungen und Anhäufung von Rundzellen. Das Peritonealepithel ist unverändert. Die Gefäße im allgemeinen groß, teilweise mit Blut gefüllt; die Gefäßwandungen auf den verschieden gefärbten Schnitten gut hervortretend. Was die elastischen Fasern betrifft, so zeigte sich nichts Auffallendes an ihnen. Sie waren überall ziemlich spärlich, in ungefähr gleichem Verhältnis vorhanden - besonders zeigte sich kein Unterschied mit der Umgebung des Risses.

Suchen wir jetzt nach einem ätiologischen Moment für die Entstehung der in Frage stehenden Ruptur, so wird uns die Anamnese, der Operationsbericht und der anatomische Befund Anhaltspunkte dafür geben können, wie sie auch in den Berichten anderer Autoren bei gleichen Situationen gefunden worden sind.

Wie aus der Krankengeschichte hervorgeht, hat Patientin kurze Zeit vor der letzten Gravidität einen Abort durchgemacht und ist im unmittelbaren Anschluß an denselben wegen Blutungen infolge von Retentio p. a. kurettiert worden. Vom Tage nach der Ausschabung an bestand hohes Fieber, viele Schüttelfröste, Erbrechen, Meteorismus, Schmerzen im Abdomen, Erscheinungen, die auf einen peritonitischen Prozeß hindeuten. Die Adhäsionen an dem durch Operation gewonnenen Präparat lassen gleichfalls erkennen, daß sich früher hier eine Peritonitis abgespielt hat. Diese wiederum, die Peritonitis, die Patientin überstand, ist wohl mit fast absoluter Sicherheit auf eine bei der Abrasio gesetzte Perforation mit der Kurette zu beziehen. Und hier, in einer früheren Verletzung der Uteruswand, hätten wir bereits die Ursache für das Platzen des Uterus im Beginne der Geburt.

$\mathrm{Baisch}^{1}$ ) hat in seiner ausführlichen Arbeit über die Zerreißung des Uterus in der Gravidität die ätiologischen Gesichtspunkte der spontanen Uterusruptur eingehend dargestellt und

1) B a is ch, Beiträge zur Geburtshilfe und Gynăkologie 1903, Bd. 7 
dabei auf die Rupturen bei Narben des Uterus nach früherem Kaiserschnitt sowohl wie auch nach früheren Verletzungen des Uterus hingewiesen.

Während die Fälle von spontanem Platzen einer Kaiserschnittsnarbe in der Schwangerschaft oder Geburt nicht so seltene Ereignisse sind ${ }^{1}$ ), finden sich Aufzeichnungen über die Spontanruptur des Uterus infolge kleiner zirkumskripter Narben in verhä1tnismäßig geringer Zahl. Es liegt dies wohl nicht an dem seltenen Vorkommen kleiner Verletzungen der Uteruswand und der eventuell daraus resultierenden Folgen in einer späteren Schwangerschaft, sondern an der geringen Beachtung dieser Momente. Gerade die durch Perforation mit der Kurette, der Sonde oder dem Dilatator herbeigeführten Verletzungen der Muskulatur können bei späteren Geburten eine große Rolle spielen. Einschlägige Fälle beweisen das:

In dem von $\mathrm{Herzfeld}$ ) mitgeteilten Falle war der Ruptur des am Ende der Gravidität befindlichen Uterus wegen atonischer Blutung post partum eine Ausschabung mit Perforation vorangegangen. In diesem Falle allerdings ist die Aetiologie nicht ganz sicher festgestellt, weil sich an dem Uterus feste Adhäsionen fanden, die die Ruptur herbeigeführt haben können.

Hon $\mathrm{ck}^{\mathbf{s}}$ ) berichtet über einen Fall von Uterusruptur am Ende der Gravidität bei einer Drittgebärenden. - Ruptur von einer Tubenecke zur andern. Ei frei im Abdomen. - Vor Eintritt dieser Schwangerschaft war bei einem Abort ein Kurettement gemacht worden.

In dem Fall von Staude) handelte es sich um eine beginnende Ruptur bei einer IV-para, bei der vier Jahre vor dieser Gravidität ein Abort mit Kurettement behandelt war. Im Anschluß daran trat eine Bauchfellentzündung ein. Staude erklärt die beginnende Ruptur so, daß bei der Ausschabung eine Perforation gemacht worden ist, die durch Serosaverklebung heilte; die Verklebung ging bei Wehenbeginn auseinander, sodaß man in der vorderen Uteruswand eine scharfrandige, runde, fünfmarkstückgroße Oeffnung palpieren konnte. Durch Kaiserschnitt wurde der Befund bestätigt.

Ganz sicher konnte $\mathrm{Baisch}^{\mathbf{5}}$ ) seinen Fall von Spontanruptur in der Gravidität auf eine Perforation des Uterus mit der Schröderschen Sonde zurückführen. Der letzten Schwangerschaft war ein Abort vorangegangen, der in der Folge starke Blutungen verursacht und eine Ausschabung des Uterus notwendig gemacht hatte. Bei dieser kam es zu einer Durchstoßung des Fundus uteri. Die Perforation war sofort bemerkt worden und heilte ohne jede Temperatursteigerung.

In unserem Falle ist das Platzen des Uterus, wenn wir den Angaben der Patientin weiter folgen, um 12 Uhr nachts eingetreten. Daß die vorhandene Narbe bereits vorher, in geringem Grade vielleicht schon in der zweiten Hälfte der Gravidität, dann aber in stärkerem Maße am Ende der Schwangerschaft und bei Beginn der Wehen, gedehnt und so zu perito. nitischen Schmerzen Veranlassung gegeben hat, geht gleichfalls aus der Krankengeschichte hervor. Die dauernd rechts sitzenden Schmerzen während der Schwangerschaft bei den Kindsbewegungen steigern sich, wie dort ersichtlich, bei Beginn und Fortschreiten der Geburt entsprechend der immer mehr zunehmenden Dehnung und dem vermehrten Innendruck.

Um 12 Uhr hatte die Patientiñ, wie erwähnt, das Gefühl, als ob in ihrem Leib etwas platze, ein Zeichen, das für die Diagnose der Ruptur charakteristisch ist. Die außerordentliche peritoneale Empfindlichkeit, die bei unserer Kranken beobachtet wurde, bildet ein weiteres Symptom für die Ruptur; und schließlich fehlte auch ein drittes Merkmal nicht, auf das immer hingewiesen wird, die Zeichen der inneren Blutung: zunehmende Blässe, kleiner, frequenter Puls, Flimmern vor den Augen, Ohnmachten, Schwindelanfälle.

Merkwürdig ist, daß eine Blutung nach außen überhaupt nicht eingetreten ist. Doch ist der Blutabgang aus der Scheide

1) Everke, Monatsschrift für Geburtshilfe und Gynäkologie Bd. 14. Krukenberg, Archiv für Gynăkologie Bd. 28. W oyer, Monatsschrift für Geburtshilfe und Gynäkologie Bd. 6. E c k s te i n, Zentralblatt für Gynäkologie 1904, No. 44. S c h n e ider, Deutsche medizinische Wochenschrift Bd. 26. G uille a u me, Zentraiblatt für Gynăkologie 1896, No. 50 . M e y e r, Zentralblatt für Gynäkologie 1903, No. 47. Couvelaire, Zentralblatt für Gynakologie 1907, S. 1517. B a is c h, Beiträge zur Ge burtshilfe und Gynäkologie 1903, Bd. 7. Ta r g e tt, Zentralblatt für Gynäkologie 1904 burtshilfe und Gynäkologie 1903, Bd. 7. T a r g e t t, Zentralblatt für Gynäkologie 1904,
No. 47. S c h i n k, Zentralblatt für Gynäkologie 1905, No. 32. J a r i n e, Zentralblatt für Gynăkologie 1905, No. 2 .

2) He r z f e ld, Zentrablatt 1901 , No. 44

3 $8 \mathrm{nck}$, Münchener medizinische Wochenschrift 1906, No. 22.

4) S ta u d e, Zentralblatt 1904, No. 22.

5) B a i s ch, Beiträge zur Geburtshilfe und Gynakologie 1903, Bd. 7 bei spontanen Uterusrupturen nach Baisch immer recht gering, er kann sogar völlig fehlen. Meyer ${ }^{1}$ ) hält einen, wenn auch meist nur geringen, so doch sehr konstanten Blutabgang aus der Scheide gleich zu Beginn der Ruptur für typisch bei der Zerreißung.

Das Kind ist bald nach Eintreten des Risses in die Bauchhöhle ausgetreten, und auch die Placenta scheint kurze Zeit später sich gelöst zu haben und in die Bauchhöhle ausgestoßen zu sein. Darauf deutet das Sistieren der Kindsbewegungen hin kurze Zeit nach der Ruptur und das baldige Schwächerwerden und allmähliche Sinken der kindlichen Herztöne.

Der Operationsbericht weist darauf hin, daß bei der vorhandenen Situation von einer konservativen Behandlung, Aneinanderbringen der Rißränder und Vernähen derselben, ab. gesehen werden mußte. Die Rißränder des fest kontrahierten Uterus klafften stark, waren zerfetzt und morsch und konnten nicht zusammengebracht werden. Ein ganz ähnlicher Befund veranlaßte $F$ üth $^{2}$ ), bei einer allerdings auf Grund einer anderen Aetiologie entstandenen Ruptur von der beabsichtigten Vernähung der Serosa Abstand zu nehmen und den Uterus supravaginal zu amputieren. Die Beschaffenheit des Risses wird, wie Baisch u. a. betonen, eine Naht desselben fast nie ermöglichen, die Amputation des Uterus in den meisten Fällen notwendig machen.

Die kindlichen Maße zeigen, daß es sich keineswegs um ein sehr großes Kind gehandelt hat. Die Beckenmessungen ergaben ein durchaus normales Verhalten. Von seiten des Kindes und des Beckens lag also kein Geburtshindernis vor.

Der mikroskopische Befund hat ein völlig negatives Resultat ergeben. Weder an den elastischen Elementen des Uterus noch an den Muskelfasern noch auch am Bindegewebe hat sich histologisch irgend etwas gefunden, was für die Ruptur verantwortlich gemacht werden könnte. Die kleinzellige Infiltration kann, wie Baisch u. a. dargelegt haben, nicht als Ursache der Ruptur hingestellt werden, und das relativ spär liche Vorhandensein von elastischen Fasern ist, wie Woltke und $\mathrm{Pick} \mathrm{k}^{3}$ ) nachgewiesen haben, ein regelmäßiger Befund am hochschwangeren Uterus. Baisch hat bereits in seiner vielfach zitierten Arbeit und in einer weiteren Publikation ${ }^{4}$ ) nachdrücklichst betont, daß die mikroskopische Untersuchung des Uterus keine wesentlichen Beiträge zur Aufklärung der Spontanrupturen in der Schwangerschaft geliefert hat und daf kleine Narben nach früheren Verletzungen sich am rupturierten Uterus nicht mehr nachweisen lassen. Unser Fall wäre demnach ein Beweis mehr für die Richtigkeit seiner Behauptungen.

Wenn auch nicht jede Narbe des Uterus zu einer Ruptur zu führen braucht, so zeigt doch vorliegender Fall, welche gefährlichen Folgen eine Perforation der Gebärmutter in einer späteren Schwangerschaft nach sich ziehen kann. Bei der Hăufigkeit der Perforationen des abortierenden Uterus mit der Kurette erscheint es angebracht, vor der Benutzung dieses Instrumentes gerade bei Ausräumung von Abortresten zu warnen in Hinsicht auf spätere Graviditäten. Ist aber eine Perforation erfolgt, so ist eine genaue Beobachtung bei einer eventuell folgenden Schwangerschaft und während der Geburt dringend erforderlich.

Zusammenfassung: Bei einer 33 jährigen Frau wurde ein Abort mit Kurettement nachbehandelt. Dabei wurde die Uteruswand perforiert. In der darauf folgenden Schwangerschaft traten starke Unterleibsschmerzen auf, ganz in der Eröffnungsperiode platzte der Uterus vom Fundus bis zum inneren Muttermund. Für die Schmerzen sowohl wie für den CorpusriB ist - nach AusschluB der sonst noch in Frage kommenden Aetiologien - die nach der Perforation entstandene Narbe verantwortlich zu machen. Der seltene Fall zeigt, wie gefährlich Narben der Gebärmutter nach früheren Verletzungen werden können und weist darauf hin, daß eine genaue Beobachtung während Schwangerschaft und Geburt nach einer Perforation dringend erforderlich ist.

1) Meyer, Beitrăge zur Geburtshilfe und Gynäkologie 1905, Bd. 9. 2) Fit th, Zentralblatt für Gynäkologie 1903, No. 9. - 3) Pick, Sammlung klinischer Vorträge N. F. No. 383 . - 4) $\mathrm{Ba}$ i s ch, Zentralblatt für Gynäkologie 1904, No. 50. 